\title{
Peripheral injury of pelvic visceral sensory nerves alters GFR $\alpha$ (GDNF family receptor alpha) localization in sensory and autonomic pathways of the sacral spinal cord
}

\author{
Shelley L. Forrest ${ }^{1 \dagger}$, Sophie C. Payne ${ }^{2}$, Janet R. Keast ${ }^{1,2 \ddagger}$ and Peregrine B. Osborne ${ }^{1,2 * \neq}$ \\ ${ }^{1}$ Pain Management Research Institute (Kolling Institute), University of Sydney at the Royal North Shore Hospital, Sydney, \\ NSW, Australia, ${ }^{2}$ Department of Anatomy and Neuroscience, The University of Melbourne, Melbourne, VIC, Australia
}

OPEN ACCESS

Edited by:

Yun-Qing Li,

The Fourth Military Medical University, China

Reviewed by:

L. Ashley Blackshaw,

University of Adelaide, Australia

David I. Hughes,

University of Glasgow, UK

*Correspondence:

Peregrine B. Osborne, Department of Anatomy and Neuroscience, The University of Melbourne, Melbourne, VIC 3010, Australia

Tel: +61 383445769

peregrine.osborne@unimelb.edu.au

tPresent address:

Shelley L. Forrest, Discipline of Pathology, University of Sydney, NSW

2006, Australia

‡These authors have contributed equally to this work.

Received: 13 January 2015 Accepted: 19 March 2015

Published: 10 April 2015

Citation:

Forrest SL, Payne SC, Keast JR and Osborne PB (2015) Peripheral injury

of pelvic visceral sensory nerves alters GFR (GDNF family receptor alpha) localization in sensory and autonomic pathways of the sacral spinal cord.

Front. Neuroanat. 9:43. doi: 10.3389/fnana.2015.00043
GDNF (glial cell line-derived neurotrophic factor), neurturin and artemin use their coreceptors (GFR $\alpha 1$, GFR $\alpha 2$ and GFR $\alpha 3$, respectively) and the tyrosine kinase Ret for downstream signaling. In rodent dorsal root ganglia (DRG) most of the unmyelinated and some myelinated sensory afferents express at least one GFR $\alpha$. The adult function of these receptors is not completely elucidated but their activity after peripheral nerve injury can facilitate peripheral and central axonal regeneration, recovery of sensation, and sensory hypersensitivity that contributes to pain. Our previous immunohistochemical studies of spinal cord and sciatic nerve injuries in adult rodents have identified characteristic changes in GFR $\alpha 1$, GFR $\alpha 2$ or GFR $\alpha 3$ in central spinal cord axons of sensory neurons located in DRG. Here we extend and contrast this analysis by studying injuries of the pelvic and hypogastric nerves that contain the majority of sensory axons projecting to the pelvic viscera (e.g., bladder and lower bowel). At $7 \mathrm{~d}$, we detected some effects of pelvic but not hypogastric nerve transection on the ipsilateral spinal cord. In sacral (L6-S1) cord ipsilateral to nerve injury, GFR $\alpha 1$-immunoreactivity (IR) was increased in medial dorsal horn and CGRP-IR was decreased in lateral dorsal horn. Pelvic nerve injury also upregulated GFR $\alpha 1$ - and GFR $\alpha 3-$ IR terminals and GFR $\alpha 1-$ IR neuronal cell bodies in the sacral parasympathetic nucleus that provides the spinal parasympathetic preganglionic output to the pelvic nerve. This evidence suggests peripheral axotomy has different effects on somatic and visceral sensory input to the spinal cord, and identifies sensory-autonomic interactions as a possible site of post-injury regulation.

Keywords: nerve injury, sacral spinal cord, dorsal horn, visceral afferents, parasympathetic nervous system, preganglionic, GDNF family of ligands (GFL), pelvic pain

\footnotetext{
Abbreviations: AOI, Area of interest; CGRP, Calcitonin gene-related peptide; DGC, Dorsal gray commissure; DRG, Dorsal root ganglion/ganglia; GDNF, Glial cell line-derived neurotrophic factor; GFR $\alpha$, GDNF family receptor alpha; LCP, Lateral collateral pathway; MCP, Medial collateral pathway; SPN, Sacral parasympathetic nucleus.
} 


\section{Introduction}

Peripheral sensory nerves are easily damaged by common surgeries, accidental trauma and disease, which can in turn cause persistent post-surgical and neuropathic pain that is difficult to treat (Costigan et al., 2009; Jensen et al., 2011; Schug, 2012). A large epidemiological study of persistent post-surgical pain has identified the location of surgical neural damage as a risk factor (Johansen et al., 2012; Schug, 2012)-as prevalence is high following surgery on the back, neck, hip and limbs, but is low after surgeries on the abdomen and pelvic viscera. This clinical finding identifies a significant limitation in preclinical studies of the relationship between nerve injury and pain, as most reports use nerve injury models where only somatic (cutaneous, muscle and joint) afferents are damaged (von Hehn et al., 2012). By far the most extensively studied models are those involving injuries to the sciatic nerve projecting to the hindlimbs or cranial nerves projecting in the neck and head. As a result, there is relatively little comparative information available on the outcome of similar injuries to major nerves that are mostly comprised of visceral sensory afferents.

Peripheral nerve injuries have complex effects on rodent primary sensory neurons (Christie and Zochodne, 2013; Scheib and Höke, 2013). It is well established that injuring peripheral axons initiates a pro-regenerative state that supports axon growth, reinnervation of target organs and recovery from sensory deficits. However, other forms of neuroplasticity expressed by primary sensory neurons after nerve injury are pathophysiological and result in persistent sensory dysfunction and pain (von Hehn et al., 2012). The effects of nerve injury are not restricted to the peripheral axons of primary sensory neurons, as central axonal projections in the spinal cord dorsal horn can also show evidence of chemical and structural neuroplasticity (Navarro et al., 2007; Yang et al., 2014). For example, sciatic nerve injury causes remodeling of the axons of peptidergic and non-peptidergic primary afferent $\mathrm{C}$-fiber axons containing calcitonin gene-related peptide (CGRP) and isolectin-B4 respectively (Bailey and Ribeiroda-Silva, 2006; Casals-Díaz et al., 2009; Keast et al., 2010). However, again these changes have mostly been characterized in the L4-L5 segments of the lumbar spinal cord using somatic nerve injury models involving different branches of the sciatic nerve.

To investigate the impact of visceral nerve injury on the spinal cord this study has transected pelvic and hypogastric nerves, which in rat are visceral nerves essential for the micturition reflex and other urogenital functions (Yoshimura, 1999; Keast, 2006). There are dramatic species differences in the organization of the sensory and autonomic components of these projection pathways but in humans the homologous nerves associated with the pelvic (inferior hypogastric) plexus are often damaged by routine surgical procedures, resulting in bladder, bowel and sexual dysfunction (Walsh and Donker, 1982; Maas et al., 2003). The pelvic nerve in rat is a mixed nerve comprising sensory $\mathrm{A} \delta$ - and C-fiber axons that input to L6-S1 spinal cord segments, parasympathetic preganglionic axons that project from L6-S1 spinal cord and function in sacral autonomic regulation, and sympathetic postganglionic axons from paravertebral ganglia (Keast, 2006). The hypogastric nerve in rat extends from the inferior mesenteric to the major pelvic ganglia. It is also a mixed nerve comprising sensory $\mathrm{A} \delta$ - and C-fiber axons that project to upper lumbar (L1/L2) spinal cord segments in rat; spinal sympathetic preganglionic axons projecting from the L1-L2 segments; as well as some sympathetic postganglionic axons projecting from the inferior mesenteric ganglion.

Very few studies have examined the impact of injury on pelvic visceral sensory neurons in any species. An early study in cats found that transecting the pelvic nerve decreased C-fibers expressing vasoactive intestinal polypeptide in the dorsal horn, but in contrast to somatic nerve injury (transection of sciatic and pudendal nerves), the peptidergic C-fibers expressing galanin and somatostatin were not affected (Anand et al., 1991). We are not aware of any equivalent studies in rodents, but our group has identified changes in these nerves after an inflammatory challenge (Forrest and Keast, 2008). Using a model of cyclophosphamide-induced bladder cystitis, we found that non-peptidergic afferents in sacral dorsal horn upregulate glial cell line-derived neurotrophic factor (GDNF) receptor alpha1 (GFR $\alpha 1)$, which is the ligand-binding receptor of GDNF (Airaksinen and Saarma, 2002). We do not know if this was due to a direct effect of the inflammatory environment or the acute structural damage of sensory terminals in the bladder that occurs in this model. However, we have also determined that GFR $\alpha$ receptors are affected by injuring peripheral sensory axons in a rat somatic nerve injury model (Keast et al., 2010). We found that sciatic nerve injury increases GFR $\alpha 1$ and the artemin receptor, GFR $\alpha 3$, in sacral spinal cord and downregulates the neurturin receptor, GFR $\alpha 2$ in lumbar cord. GFR $\alpha 1$ and GFR $\alpha 2$ are expressed in separate populations of nonpeptidergic C-fiber afferents, GFR $\alpha 1$ is also expressed in some myelinated afferents, and GFR $\alpha 3$ is exclusively expressed in a sub-population of peptidergic C-fibers (Orozco et al., 2001; Kalous et al., 2007, 2009; Ernsberger, 2008; Keast et al., 2010). On this basis, our aim here was to determine if axotomy of peripheral visceral axons in the pelvic or hypogastric nerves in rat have similar or different effects on the spinal cord distribution of four distinct populations of afferent fibers identified with GFR $\alpha 1$, GFR $\alpha 2$, GFR $\alpha 3$ and CGRP (which includes the GFR $\alpha 3$-positive population). We also investigated if these injuries had any effect on GFR $\alpha$ expression in neuronal somata of the sacral parasympathetic nucleus (SPN), an area containing preganglionic neurons would be axotomised by pelvic or hypogastric nerve transection.

\section{Methods}

\section{Animals and Surgical Procedures}

All procedures complied with the Australian Code for the Care and Use of Animals for Scientific Purposes (National Health and Medical Research Council of Australia) and were approved by Animal Ethics Committees at the University of Sydney or the University of Melbourne. Adult male Sprague-Dawley rats (20 in 
total, aged 6-8 weeks; Animal Resources Centre, Murdoch, WA) were housed in groups of 3 , under a $12 \mathrm{~h}$ light-dark cycle, with ad libitum access to water and standard chow.

All surgical procedures were performed under isoflurane anesthesia $(3 \%$ in oxygen for induction, $1.5-2 \%$ for maintenance). Four types of nerve injury surgeries (unilateral transection of the pelvic $(n=11)$ or hypogastric $(n=4)$ nerves; bilateral transection of both the pelvic and hypogastric $(n=3)$ nerves or pelvic nerve only $(n=2))$ were performed using published methods (Kalous and Keast, 2010; Peddie and Keast, 2011). In brief, the lower abdominal cavity was opened via a midline excision to expose the pelvic organs, which were displaced to access the pelvic ganglia located on the dorsolateral aspect of the prostate gland. The hypogastric and/or pelvic nerves were then isolated from underlying prostate tissue with fine forceps and cut with iris scissors at approximately $1 \mathrm{~mm}$ from the ganglion, after which the abdominal muscle and skin were sutured. All rats were closely monitored following surgery. As bilateral transection of the pelvic nerve prevents micturition, animals that received this surgery had their bladder emptied manually at intervals of less than $12 \mathrm{~h}$.

\section{Tissue Preparation}

Seven days after surgery, rats were deeply anesthetized with sodium pentobarbitone $(80 \mathrm{mg} / \mathrm{kg}$ i.p.) and transcardially perfused with $0.9 \%$ saline containing $1.25 \%$ sodium nitrite and $0.036 \%$ heparin, followed by freshly made $4 \%$ paraformaldehyde fixative in $0.1 \mathrm{M}$ phosphate buffer ( $\mathrm{PB}, \mathrm{pH}$ 7.4). Spinal cords were removed and post-fixed overnight in the same fixative at $4^{\circ} \mathrm{C}$, then washed in $0.1 \mathrm{M}$ phosphate buffered saline (PBS, $\mathrm{pH}$ 7.2) and stored at $4^{\circ} \mathrm{C}$ in PBS containing $0.1 \%$ azide. Fixed spinal cords were marked by a superficial cut to the ventrolateral gray matter to identify the side ipsilateral to nerve injury and segmented into sacral (L6-S1) and upper lumbar (L1-2) regions. They were then cryoprotected overnight in PBS containing $30 \%$ sucrose and cut on a cryostat. Transverse sections $(40 \mu \mathrm{m})$ were collected in a 1 in 4 series so that sections processed for the same substance were sampled at least $160 \mu \mathrm{m}$ apart. To visualize the rostrocaudal extent of the SPN, horizontal sections $(40 \mu \mathrm{m})$ of the L4-S2 segment were also collected in a 1 in 2 series so that sections processed for the same substance were sampled at least $80 \mu \mathrm{m}$ apart.

\section{Chromagen Immunohistochemistry}

All sections were processed free-floating using a glucose oxidase/nickel enhanced diaminobenzidine (DAB) method (Hamlin et al., 2007; Kalous et al., 2007, 2009). In brief, sections were washed in $\mathrm{PB}$ prior to incubations (30 $\mathrm{min}$ ) in $50 \%$ ethanol, $50 \%$ ethanol containing $3 \% \mathrm{H}_{2} \mathrm{O}_{2}$ to block endogenous peroxidase activity, and a blocking solution of $5 \%$ normal horse serum (NHS) in PB. Transverse sections were then incubated on a shaker ( $48 \mathrm{~h}$ at room temperature) in affinity purified antisera raised against goat GFR $\alpha 1$ (1:400; R\&D Systems, Minneapolis, $\mathrm{MN}$, cat. no. AF560; RRID:AB_2110307), GFR $\alpha 2$ (1:1000, R\&D Systems, cat. no. AF429; RRID:AB_2294621), GFR $\alpha 3$ (1:300, R\&D Systems, cat. no. AF2645; RRID:AB_2110295) or rabbit CGRP (1:2000, Millipore, Vic, Australia, cat. no. RRID:AB_2068655). Details of antibody characterization are provided in previous reports (Kalous et al., 2007, 2009; Keast et al., 2010; Forrest et al., 2013, 2014) and additional references linked to their research resource identifiers. ${ }^{1}$ Horizontal sections were incubated in GFR $\alpha 1$ or GFR $\alpha 3$ antisera only. After washes in $\mathrm{PB}$, sections were then incubated overnight in biotin-conjugated anti-goat or anti-rabbit affinity purified antisera (1:1000, Jackson ImmunoResearch Laboratories, West Grove, PA) raised in donkey. All antisera dilutions were made using $\mathrm{PB}$ containing $2 \%$ NHS and $0.2 \%$ triton $\mathrm{X}-100$.

Following washes in $\mathrm{PB}$, sections were then incubated $(2 \mathrm{~h}$ at room temperature $)$ in avidin-biotin complex $(6 \mu \mathrm{l} / \mathrm{ml}$, Vectastain Elite kit; Vector Laboratories, Burlingame, CA), followed by a 15 min incubation in a nickel DAB solution (2\% nickel sulfate, $0.2 \%$ D-glucose, $0.04 \%$ ammonium chloride, and $0.025 \% \mathrm{DAB}$ in $0.1 \mathrm{M}$ sodium acetate buffer $\mathrm{pH} 6.0$ ). Glucose oxidase $(0.02 \%)$ was then added to obtain black chromagen staining, which was monitored using a dissecting microscope and stopped by washing in large volumes of acetate buffer. Sections were mounted from $0.9 \%$ saline onto slides $(0.1 \%$ gelatinized $)$, dried overnight, and dehydrated in ascending concentrations of ethanol prior to being cleared in histolene, and cover slipped with DPX water-free mounting media (Crown Scientific, Mulgrave, VIC, Australia).

\section{Image Analysis and Optical Density Measurements}

The effect of unilateral pelvic or hypogastric nerve transection on optical density of immunohistochemical chromagen staining within the dorsal horn and SPN was measured by image analysis (Kalous et al., 2007, 2009; Keast et al., 2010). Figure 1 shows the three areas of interest (AOIs) used for this analysis, which were in accordance with our previous study of the effect of experimental cyclophosphamide-induced cystitis on GFR $\alpha$-immunoreactivity (IR) in sacral spinal cord (Forrest and Keast, 2008). Images were captured under a $10 \times$ objective with an Olympus BX51 microscope (Olympus Australia, Melbourne, Vic) and a Zeiss AxioCam HRc camera controlled by Axiovision 4.2 software (Carl Zeiss, Australia), and saved as 8 -bit monochrome $(1300 \times 1030$ pixels $)$ TIFF files. The microscope illumination and camera acquisition settings were the same for all images, and were set to maximize the dynamic range with no saturated pixels (Kalous et al., 2007, 2009; Keast et al., 2010).

The AOIs in the medial and lateral dorsal horn and SPN on the ipsi- and contralateral side of the same section. For quantitative analysis of immunostaining intensity, images ipsiand contralateral to injury were taken from six randomly selected lumbar and sacral sections. Optical density was assessed in areas of interest (AOIs, $130 \times 130 \mu \mathrm{m}$; Figure 1, boxes 1 and 2 ) in the dorsal horn and in the SPN (circular AOI, diameter $=250 \mu \mathrm{m}$; Figure 1, circle 3), demonstrated in Figure 1. AOI boxes 1 and 2 were used to assess immunostaining intensity of each antibody in the dorsal horn, but AOI circle 3 was used to assess

\footnotetext{
${ }^{1}$ antibodyregistry.org
} 


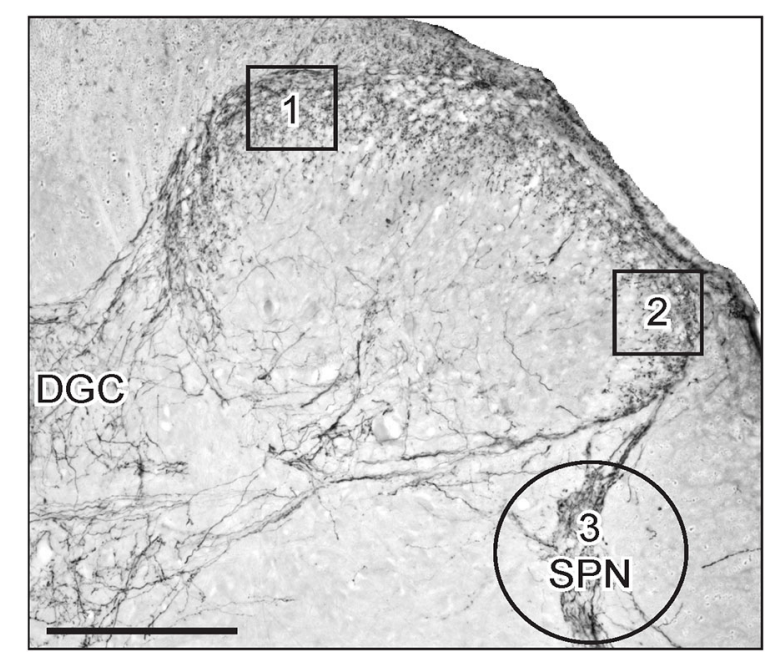

FIGURE 1 | Areas of interest (AOIs) used for densitometric image analysis following visceral nerve transection. Image shows a micrograph of CGRP-immunoreactivity (IR) in the superficial dorsal horn and sacral parasympathetic nucleus (SPN) within sacral spinal cord. AOls 1 and 2 (130 × $130 \mu \mathrm{m}$ ) were aligned with the medial and lateral margins of the superficial dorsal horn (boxes 1 and 2, respectively). Both AOls were assessed for GFR $\alpha 1$, GFR $\alpha 2$, GFR $\alpha 3$ and CGRP in the lumbar and sacral spinal cord. The AOI encompassing the SPN (circle 3, diameter $250 \mu \mathrm{m}$ ) was assessed for GFR $\alpha 1$, GFR $\alpha 3$ and CGRP. Image also shows location of the dorsal gray commissure (DGC). Scale bar represents $200 \mu \mathrm{m}$

immunostaining intensity for GFR $\alpha 1$, GFR $\alpha 3$ and CGRP in the SPN. The circular AOI was large enough to include neurons and fibers within the SPN and afferent fibers that terminated dorsal to the SPN. The optical density value of an antibody was obtained from all six sections and averaged to obtain a single value for each AOI per animal. For each antibody, the mean optical density value of the white matter was subtracted. The resultant changes in optical density could reflect changes in the staining intensity of fibers or fiber density within the AOI. Changes in optical density were equated to changes in staining intensity of fibers or changes in fiber density within the AOI.

\section{Neuronal Counts}

GFR $\alpha 1$-IR neurons were counted in the SPN on the ipsi- and contralateral sides after unilateral transection, and on one side after bilateral transection. GFR $\alpha 1$-positive neurons were counted in 5 horizontal spinal cord sections and data expressed as the mean \pm SEM of the total number of neurons per section with no stereological correction.

\section{Statistics and Figure Production}

All data are expressed as the mean \pm SEM and $P<0.05$ were regarded as statistically significant. Statistical analyses were performed using SPSS v16 for Mac (Chicago, IL) or GraphPad Prism 5.0a (GraphPad Software, La Jolla, CA). Differences between sides of GFR $\alpha 1-3$ and CGRP immunostaining in the medial and lateral dorsal horn and SPN were analyzed using paired $t$-tests. For figure production, minor adjustments were made to brightness and contrast to best represent the immunostaining as viewed directly under the microscope (Adobe InDesign and Photoshop CS2; Adobe Systems, San Jose, CA, USA).

\section{Results}

We examined the patterns of neuronal IR in regions of the rat sacral (L6/S1)(Figure 1) and upper lumbar (L1-L2) spinal cord that receive sensory input from the pelvic and hypogastric nerves, respectively. Transganglionic tracing studies in rat (Morgan et al., 1986; Nadelhaft and McKenna, 1987) show the sensory input at both levels distributes in Lissauer's tract, superficial and deep dorsal horn, medial (MCP) and lateral collateral pathways (LCP), lamina $\mathrm{X}$ and the dorsal gray commissure (DGC; Figure 1). In sacral cord, there are also terminal arborizations in the sacral parasympathetic nucleus (SPN; Figure 1), which contains the parasympathetic preganglionic autonomic neurons that project in the pelvic nerve (Hancock and Peveto, 1979; Morgan et al., 1981; Nadelhaft and Booth, 1984).

To determine if unilateral lesions of the pelvic or hypogastric nerves affect the central axons of dorsal root ganglia (DRG) neurons we used digital image analysis to compare areas of interest (AOIs) in the spinal cord contralateral and ipsilateral to the injury. GFR $\alpha 1$ - and GFR $\alpha 2$-IR were used as markers for the terminals of non-peptidergic C-fiber afferents (Forrest and Keast, 2008) whereas CGRP- and GFR $\alpha 3$-IR were used as markers for the terminals of peptidergic C-fiber afferents (Forrest and Keast, 2008; Keast et al., 2010). The distribution of all markers in the dorsal horn of the sacral (L6/S1) and lumbar (L1/2) spinal cord (Figures 2, 4-6) contralateral to both injuries was broadly consistent with previous reports (Forrest and Keast, 2008; Keast et al., 2010). However, as the experimental design was limited to a within-subject comparison of the contralateral and ipsilateral spinal cord, this precluded using quantitative analysis to detect an effect of nerve injury on the contralateral dorsal horn. Mean optical density measurements from three AOIs in medial and lateral superficial dorsal horn and SPN are summarized in Table 1 for each type of IR, spinal cord region and nerve injury group studied in the experiment.

\section{GFR $\alpha 1$ Immunoreactivity}

GFR $\alpha 1$-IR terminals in the spinal cord contralateral to nerve injury (sacral: Figures 2A,E lumbar: Figures 2C,G) were distributed primarily in lamina II (outer) of the dorsal horn as previously described (Forrest and Keast, 2008; Kalous et al., 2009). Occasional weakly stained fibers also projected in the LCP and DGC, an area that contains autonomic preganglionic axons and interneurons (Morgan et al., 1986). In sacral cord, weakly stained terminal arborizations and some neuronal cell bodies were also present in the SPN. GFR $\alpha 1$-IR motor neurons were also found in the ventral horn (not shown).

Optical density measurements of GFR $\alpha 1-I R$ in the sacral spinal cord 7 days after unilateral pelvic nerve transection (Table 1; Figures 2A,B,M) detected a significant increase in the medial superficial dorsal horn whereas in the lateral 

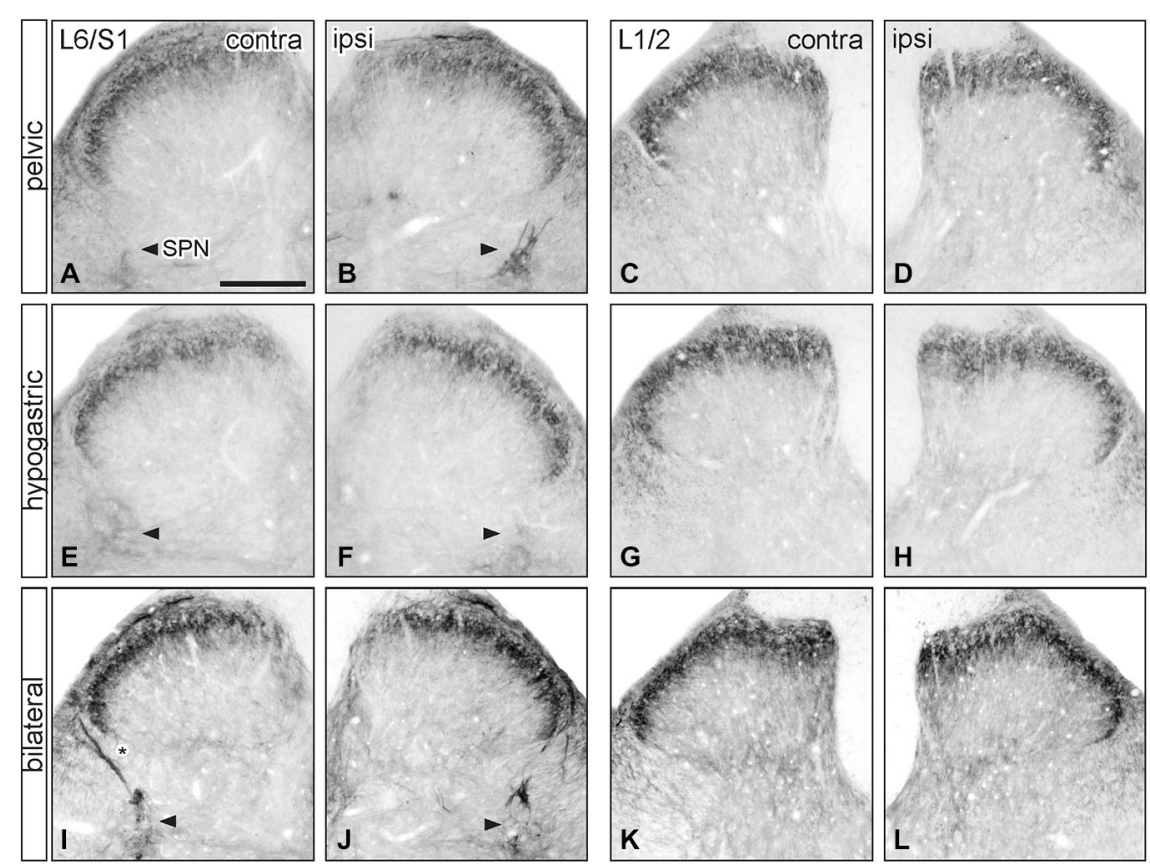

M Pelvic nerve transection
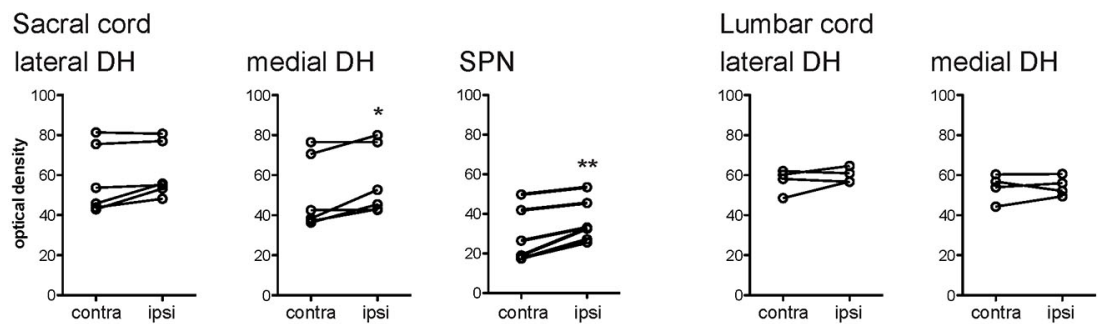

N Hypogastric nerve transection
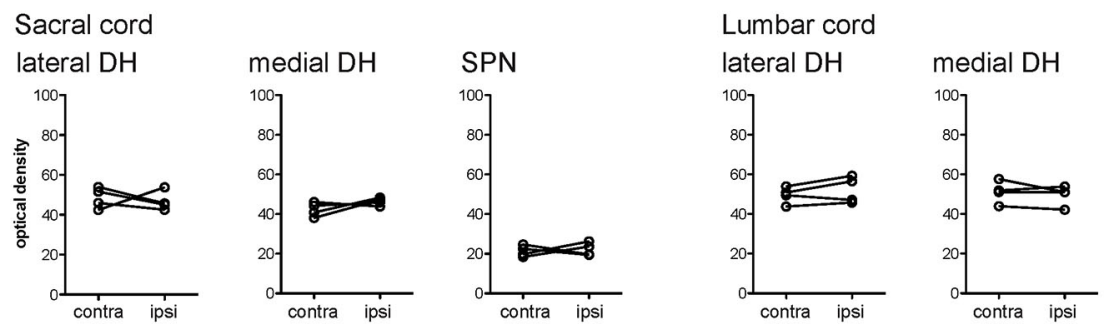

FIGURE 2 | Distribution of GFR $\alpha 1-$ IR in sacral and lumbar spinal cord following visceral nerve transection. For each type of injury, images were taken from left and right sides of the same section, and transverse segments of each spinal level from the same animal. (A-L) Images show GFR $\alpha 1-I R$ in sacral (L6/S1) and lumbar (L1/2) spinal cord at 7 days after unilateral pelvic nerve transection (A-D), unilateral hypogastric nerve transection $(\mathbf{E}-\mathbf{H})$ or bilateral pelvic and hypogastric nerve transection (I-L). (M) Quantification of GFR $\alpha 1$ optical density following unilateral pelvic nerve transection shows that in sacral cord there was no effect of injury in lateral dorsal horn, whereas a small but significant increase of GFR 1 -IR was observed in the medial dorsal horn and sacral parasympathetic nucleus (SPN) ipsilateral to injury. In lumbar spinal cord, no effect of injury on GFR $\alpha 1$ IR was detected. (N) Hypogastric nerve transection had no effect on GFR $\alpha 1$-IR in sacral or lumbar spinal cord. Data represents the mean \pm SEM ( $n=6$ rats for sacral data following unilateral pelvic nerve transection, $n=4$ rats for lumbar data following unilateral pelvic and hypogastric nerve injury) and was analyzed using a paired $t$-test. The SPN in sacral cord is indicated with arrowheads (A,B,E,F,I,J). Scale bar in A applies to all images and represents $200 \mu \mathrm{m}$. dorsal horn the difference approached borderline significance $(P=0.07, n=6)$. A significant increase in GFR $\alpha 1$-IR was also detected in the area of the SPN. No effect of injury on GFR $\alpha 1-I R$ was detected in lumbar spinal cord (Table 1;
Figures 2C,D,M), and no change was detected at either spinal level after unilateral hypogastric nerve transection (Table 1; Figures $2 \mathrm{E}-\mathbf{H}, \mathbf{N})$. Consistent with the increase in GFR $\alpha 1-I R$ measured quantitatively in the SPN, we observed that staining in 

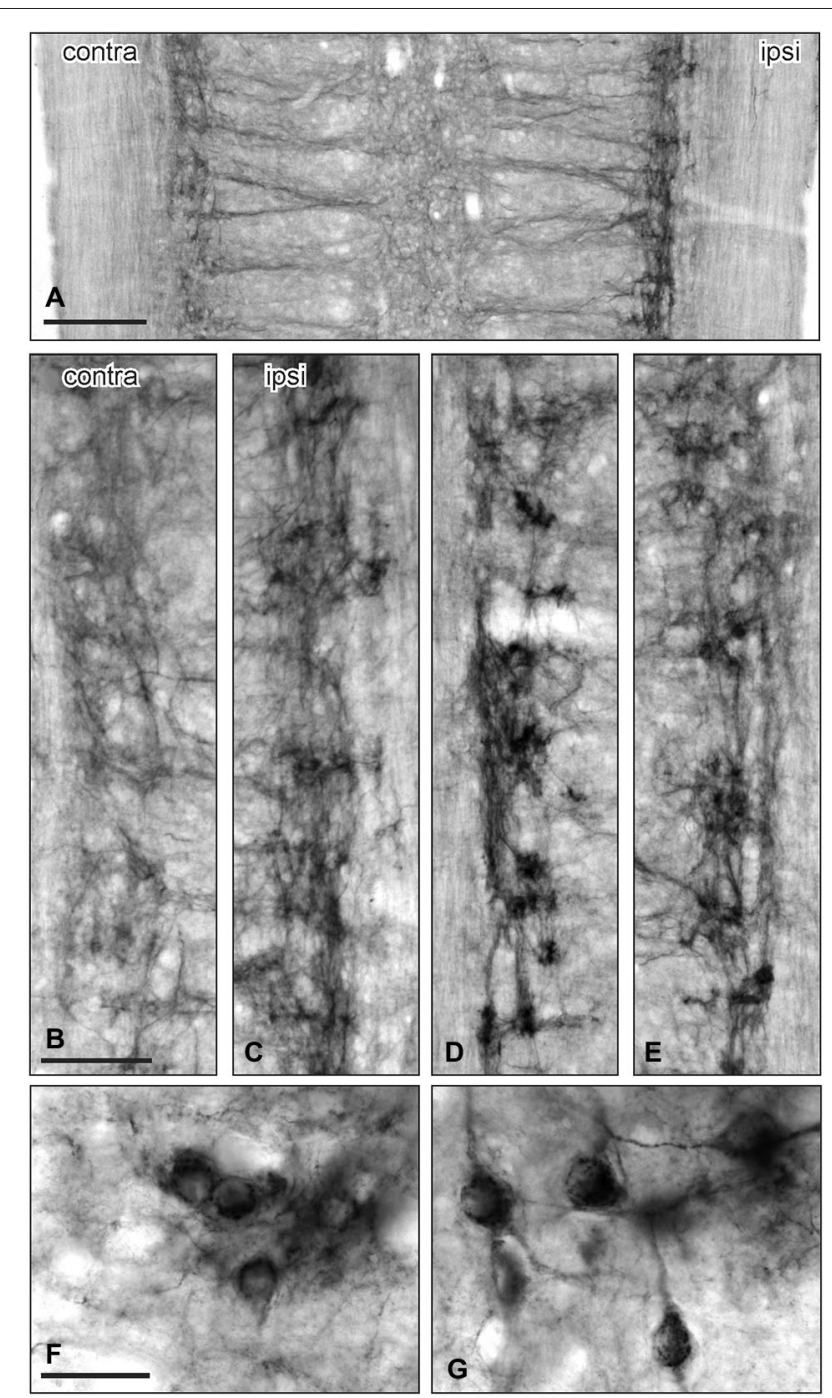

FIGURE 3 | GFR $\alpha 1-$ IR in the sacral parasympathetic nucleus (SPN) viewed in horizontal spinal cord sections after pelvic nerve injury. (A-C): Asymmetric upregulation of GFR $\alpha 1-I R$ in the SPN ipsilateral to a unilateral pelvic nerve transection. (D,E) Symmetric upregulation of GFR $\alpha 1-I R$ in the SPN on both sides after bilateral pelvic nerve injury. $\mathbf{( F , G ) ~ E x a m p l e s ~ o f ~}$ GFR $\alpha 1-I R$ somata in SPN ipsilateral to pelvic nerve transection. Scale bars represent $200 \mu \mathrm{m}$ (A), $50 \mu \mathrm{m}$ (B-E), and $50 \mu \mathrm{m}$ (F,G)

this region was also intense and appeared to occupy a larger area in sections taken from rats that underwent joint bilateral pelvic and hypogastric nerve transections (Figures 2I-L). No further differences were identified by visual inspection of these sections.

The above observations suggested that a major effect of unilateral pelvic nerve transection was to increase GFR $\alpha 1$-IR in the SPN, a region that contains autonomic preganglionic neurons that project in the pelvic nerve (Hancock and Peveto, 1979; Morgan et al., 1981; Nadelhaft and Booth, 1984). We made further qualitative observations relating to this result by viewing the rostro-caudal extent of the SPN in horizontal spinal cord sections. Seven days after unilateral pelvic nerve transection GFR $\alpha 1$-IR fibers, and neuronal cell bodies in the SPN, were more intensely stained on the ipsilateral side (Figures $\mathbf{3 A - C}$ ). The somata were distributed throughout the rostro-caudal extent of the SPN and were commonly observed in small clusters of up to 4-6 neurons (Figures 3F,G). More neurons were counted on the side ipsilateral to the injury (total count/section from 5 sections per rat: ipsilateral: $74.1 \pm 5.3$ and contralateral: $5.1 \pm$ 1.8; $P=0.0002, n=5)$. This asymmetric distribution of GFR $\alpha 1$-IR was not observed after bilateral nerve transection (Figures 3D,E), which resulted in more intense staining on both sides of the spinal cord.

\section{GFR $\alpha 2$ Immunoreactivity}

GFR $\alpha 2$-IR fibers in the spinal cord contralateral to nerve injury were primarily distributed in lamina II (inner) of the dorsal horn (sacral: Figures 4A,E; lumbar: Figures 4C,G) as previously described (Kalous et al., 2007; Forrest and Keast, 2008; Keast et al., 2010). Weakly stained fibers also projected in the DGC in occasional sections but were not found in the LCP or SPN, and no GFR $\alpha 2$-IR neuronal cell bodies were identified.

Optical density measurements of GFR $\alpha 2$-IR in the sacral spinal cord 7 days after unilateral pelvic nerve transection showed no effect of injury in the lateral or medial dorsal horn (Table 1, Figures 4A,B,M). However, in the lumbar spinal cord a small but significant reduction in GFR $\alpha 2$-IR was detected in the medial but not the lateral dorsal horn (Table 1, Figures $4 \mathrm{C}, \mathrm{D}, \mathbf{M})$. No change in GFR $\alpha 2$-IR was detected at either spinal level after unilateral hypogastric nerve transection (Table 1, Figures $4 \mathrm{E}-\mathrm{H}, \mathbf{N}$ ). No differences were identified by visual inspection in sacral or lumbar spinal cord after bilateral nerve transection (Figures 4I-L).

\section{CGRP Immunoreactivity}

CGRP-IR fibers in the spinal cord contralateral to nerve injury (sacral: Figures 5A,E; lumbar; Figures $\mathbf{5 C , G}$ ) were primarily confined to lamina I and II and deeper laminae in the dorsal horn as previously described (Carlton et al., 1988; Lawson et al., 1993; Kalous et al., 2009). In sacral spinal cord, CGRP-IR fibers in the superficial laminae extended along the medial border of the dorsal horn and in some sections these fibers formed a continuous band following the edge of gray matter to the medial side of the opposite dorsal horn. Many CGRP-IR fibers were also present in the DGC (not shown), LCP and associated with the SPN (Figures 5A,E). In lumbar spinal cord, CGRP-IR fibers also extended along the medial border of the dorsal horn but were not as prevalent as those in sacral levels. At this spinal level, CGRPIR fibers were not associated with the IML. At both spinal levels, CGRP-IR motor neurons were present in the ventral horn (not shown). Neuronal CGRP-IR somata were not observed in any other region.

Optical density measurements of CGRP-IR in the sacral dorsal horn 7 days after unilateral pelvic nerve transection identified a significant but small decrease in the lateral but not medial superficial dorsal horn (Table 1, Figures 5A,B,M). No effect of unilateral pelvic nerve injury was detected in the SPN (Table 1, Figure $5 \mathrm{M}$ ) or in lumbar spinal cord (Table 1, Figures 5C,D,M). Quantification of CGRP immunostaining in sacral and lumbar spinal cord following unilateral hypogastric 

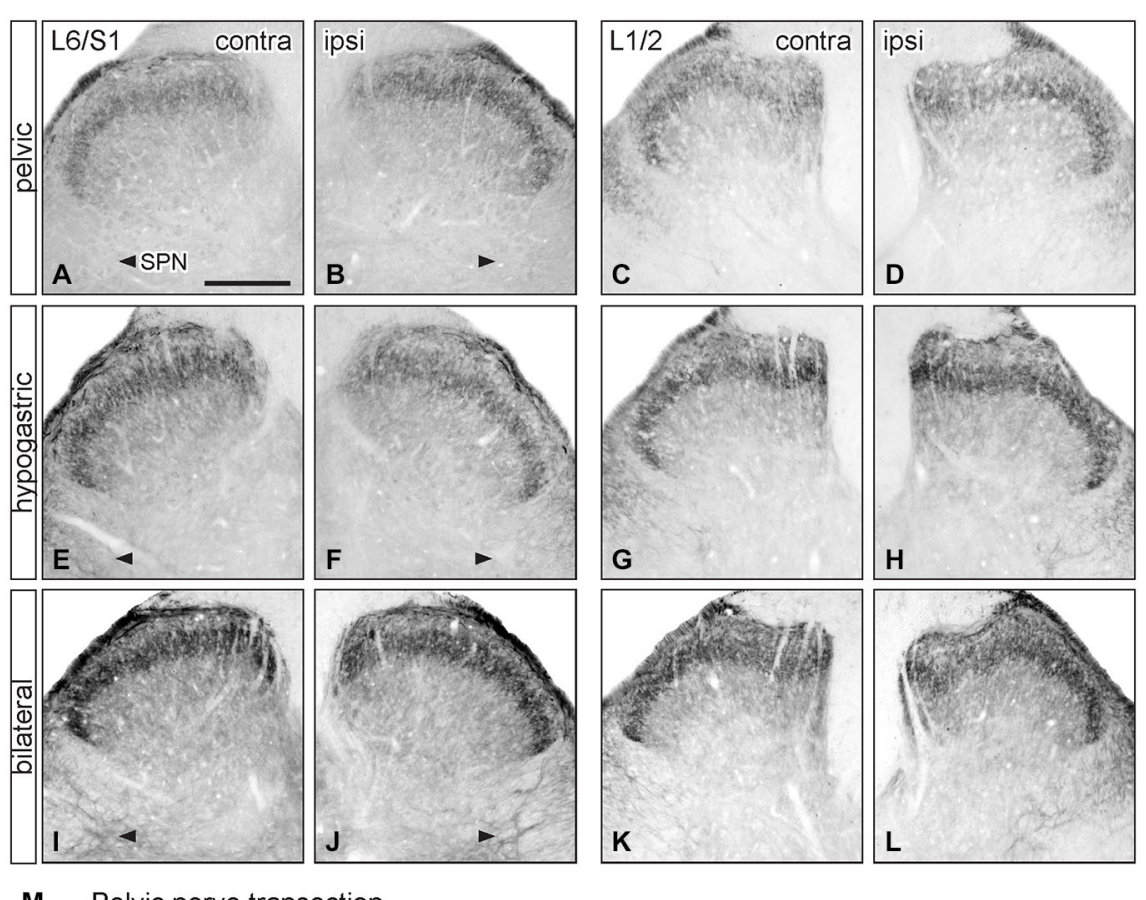

M Pelvic nerve transection
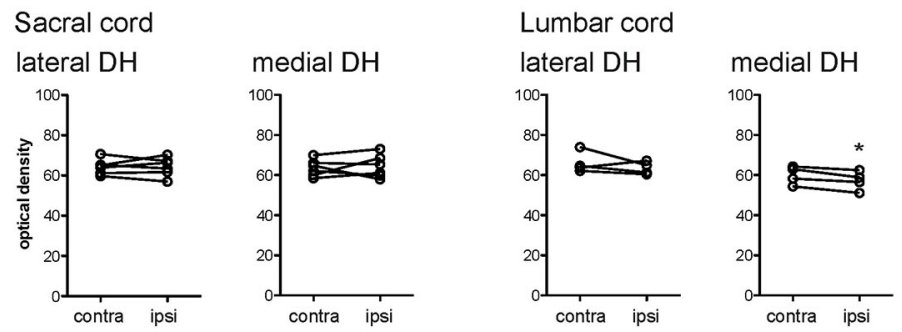

N Hypogastric nerve transection
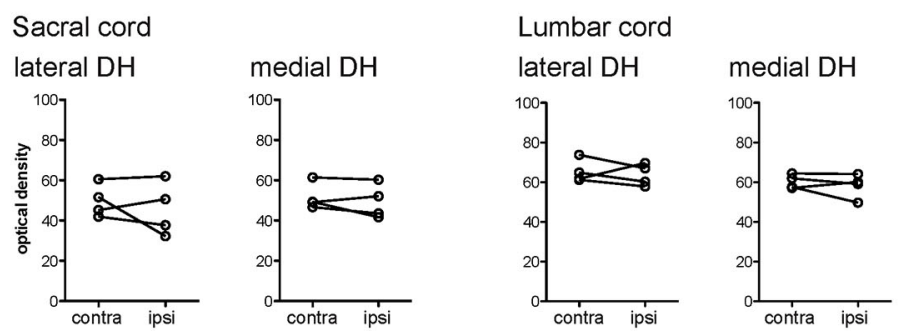

FIGURE 4 | Distribution of GFR $\alpha 2$-IR in sacral and lumbar spinal cord following visceral nerve transection. For each type of injury, images were taken from left and right sides of the same section, and transverse segments of each spinal level from the same animal. (A-L) Images show GFR $\alpha 2-I R$ in sacral (L6/S1) and lumbar (L1/2) dorsal horn at 7 days after unilateral pelvic nerve transection (A-D), unilateral hypogastric nerve transection $(\mathbf{E}-\mathbf{H})$ or bilateral pelvic and hypogastric nerve transection (I-L). (M) Following unilateral pelvic nerve transection, no change in GFR $\alpha 2$-IR was seen in sacral dorsal horn. In lumbar spinal cord, there was a small but significant decrease in GFR $\alpha 2-$ IR only in the medial dorsal horn. (N) Unilateral hypogastric nerve transection had no effect on GFR $\alpha 2-$ IR in sacral or lumbar dorsal horn. Data represents the mean \pm SEM ( $n=6$ rats for sacral data following unilateral pelvic nerve transection, $n=4$ rats for lumbar data following unilateral pelvic and hypogastric nerve transection) and was analyzed using a paired $t$-test. Scale bar represents $200 \mu \mathrm{m}$. nerve transection showed no effect of injury on CGRP-IR at either spinal level (Table 1, Figures 5E-H,N). Following combined bilateral pelvic and hypogastric nerve transections, no further differences were identified by visual inspection in the distribution of CGRP-IR at either spinal level (Figures 5I-L).

\section{GFR $\alpha 3$ Immunoreactivity}

GFR $\alpha 3$-IR in spinal cord dorsal horn is almost exclusively colocalized in the terminals of a major subpopulation of CGRPIR peptidergic afferent neurons (Orozco et al., 2001; Keast et al., 2010). GFR $\alpha 3$-IR fibers in the spinal cord contralateral to 

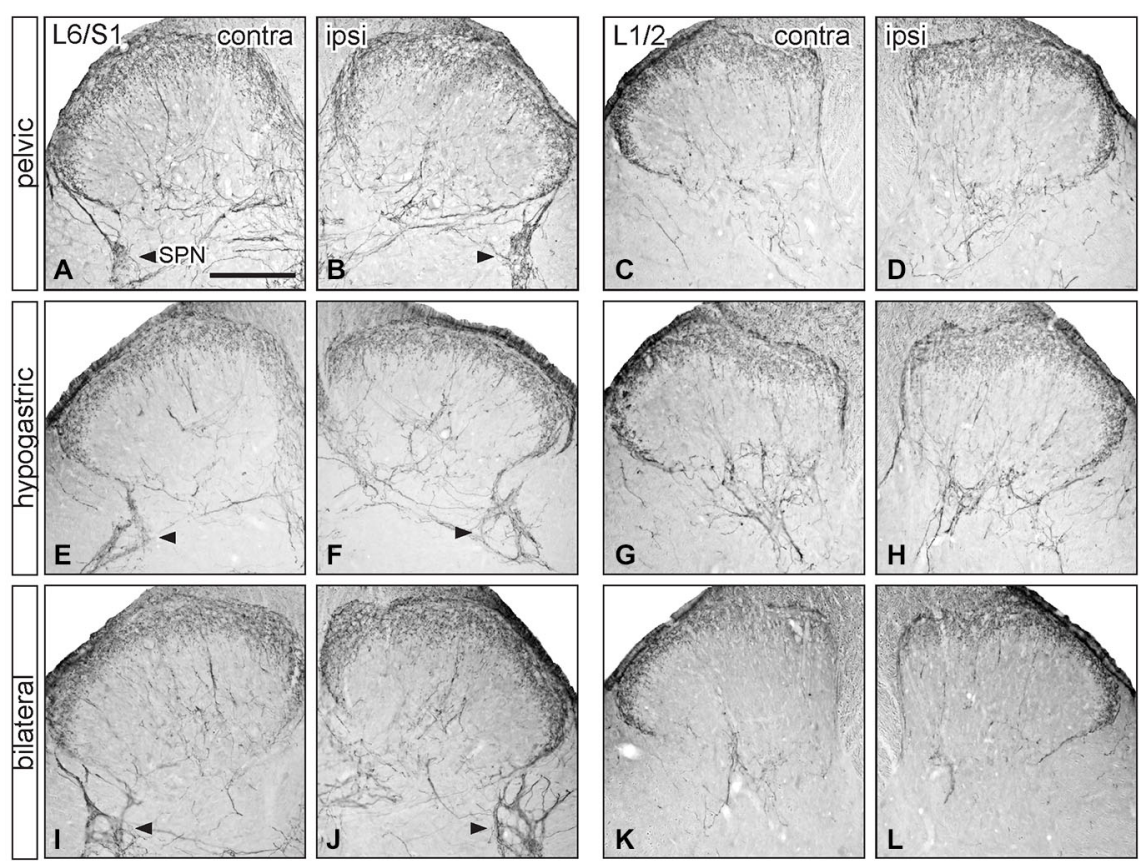

M Pelvic nerve transection

Sacral cord
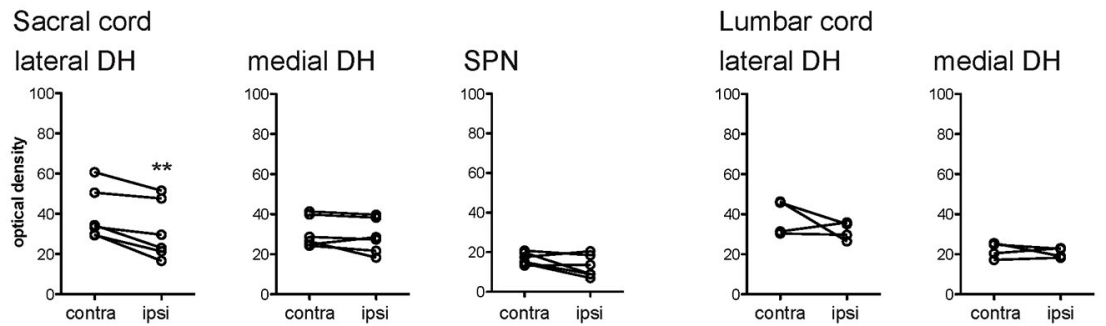

N Hypogastric nerve transection
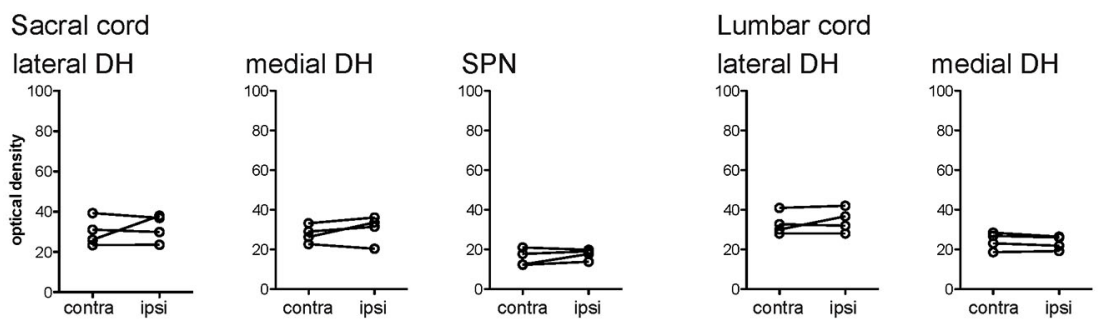

FIGURE 5 | Distribution of CGRP-IR in sacral and lumbar spinal cord following visceral nerve transection. For each type of injury, images were taken from left and right sides of the same section, and transverse segments of each spinal level from the same animal. (A-L) Images show CGRP-IR in sacral (L6/S1) and lumbar (L1/2) dorsal horn 7 days after unilateral pelvic nerve transection (A-D), unilateral hypogastric nerve transection $(\mathbf{E}-\mathbf{H})$ or bilateral pelvic and hypogastric nerve transection (I-L). (M) Following unilateral pelvic nerve transection, optical density analysis in sacral cord showed CGRP-IR decreased in only in the lateral dorsal horn. In lumbar cord, there was no change in CGRP-IR. (N) Hypogastric nerve transection had no effect on CGRP-IR in sacral or lumbar spinal cord. Data represents the mean $\pm \operatorname{SEM}(n=6$ rats for sacral data following unilateral pelvic nerve transection, $n=4$ rats for lumbar data following unilateral pelvic hypogastric nerve transection) and was analyzed using a paired t-test. SPN (L6/S1) is indicated with arrowheads (A,B,E,F,I,J). Scale bar represents $200 \mu \mathrm{m}$. nerve injury (sacral: Figures 6A,E; lumbar: Figures 6C,G) were distributed primarily in lamina I of the dorsal horn, consistent with previous studies (Forrest and Keast, 2008; Kalous et al., 2009; Keast et al., 2010). In sacral spinal cord, GFR $\alpha 3$-IR fibers projected in LCP and DGC and terminal arborizations were present in the SPN. In some sections, GFR $\alpha 3$-IR fibers in the superficial laminae extended along the medial border of the dorsal horn and formed a continuous band that extended to the opposite dorsal horn. GFR $\alpha 3$-IR fibers were not observed in the LSN. In lumbar spinal cord, GFR $\alpha 3$-IR fibers had a 
more restricted distribution and were primarily localized to lamina I (Figures 6C,G). No GFR $\alpha 3$-IR neuronal cell bodies were observed in either level of the spinal cord.

Optical density measurements of GFR $\alpha 3$-IR in the sacral spinal cord 7 days after unilateral pelvic nerve transection showed no effect of injury in the lateral or medial superficial dorsal horn but did detect a significant increase in the area of the SPN (Table 1, Figures 6A,B,M, 7A-C). No effect of injury was detected in lumbar spinal cord (Table 1, Figures 6C,D,M). Quantitation of GFR $\alpha 3$-IR in the sacral and lumbar superficial dorsal horn 7 days after unilateral hypogastric nerve transection showed no effect of injury at either spinal level (Table 1, Figures 6E-H,N). Consistent with the increase in GFR $\alpha 3-I R$ measured quantitatively in the SPN, we also observed that staining in this region was also intense in sections taken from rats that underwent joint bilateral pelvic and hypogastric nerve transections (Figures 6I-L) and closely resembled the side ipsilateral to unilateral pelvic nerve transection.

GFR $\alpha 3$-IR in the SPN was also examined in horizontal sections taken from rats that underwent unilateral pelvic or bilateral nerve transections. GFR $\alpha 3$-IR fibers were more intensely stained on the side ipsilateral to unilateral pelvic nerve transection but there were no immunoreactive neuronal cell bodies (Figures 7B,C). GFR $\alpha$ 3-IR fibers also appeared more intensely stained in the SPN after bilateral pelvic nerve transection (Figures 7D,E), but this was not quantified. GFR $\alpha 3$ IR fibers in the SPN formed tight nests and bundles of fibers (Figures 7F,G).

\section{Discussion}

The primary aim of this study was to determine how injuries to two major visceral nerves, the pelvic and hypogastric nerves, affect the central terminals of specific populations of sensory axons in sacral or upper lumbar spinal cord. In rat, these spinal levels are responsible for sensory and autonomic regulation of pelvic organs. We have previously found that damaging peripheral somatic sensory fibers by transecting the sciatic nerve causes extensive remodeling of their central axons in the dorsal horn of the rat spinal cord. This was detected immunohistochemically by increases in the markers GFR $\alpha 1$ or GFR $\alpha 3$ and decreases in GFR $\alpha 2$ but no change detected in CGRP (Keast et al., 2010). Using the same markers, this study has found the effects of injuring visceral nerves are less extensive. GFR $\alpha 1$-IR, a marker for a class of the non-peptidergic type of C-fiber afferent and also expressed in some myelinated afferents, was increased in the medial dorsal horn by pelvic nerve injury, and there was a small reduction in CGRP-IR, a marker of peptidergic C-fiber afferents, in the lateral dorsal horn. It is possible that in comparison to the sciatic C-fiber afferent input to lumbar dorsal horn the contribution made by pelvic and hypogastric nerves is smaller, making it more difficult to detect post-injury changes. However, we would expect this to be at least partly offset by the far more extensive projections of central visceral C-fiber axons within the spinal cord (Sugiura et al., 1989, 1993). Furthermore, we have previously reported that widespread upregulation of GFR $\alpha 1-$ IR can be detected in sacral dorsal horn

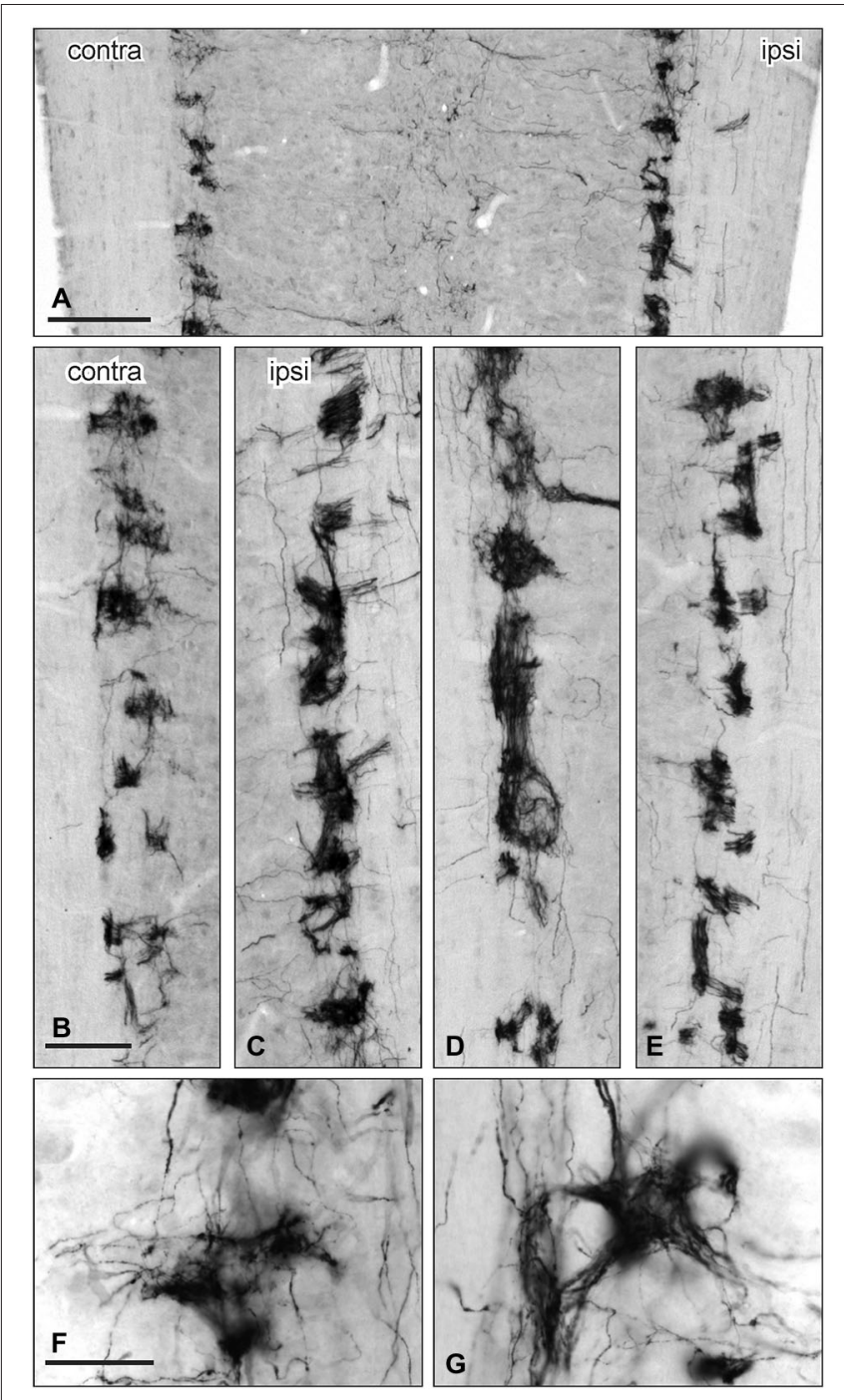

FIGURE 7 | Distribution of GFR $\alpha 3$-IR in the sacral parasympathetic nucleus (SPN) viewed in horizontal sections following pelvic nerve transection. (A-C) Asymmetric upregulation of GFR $\alpha 3-I R$ in the SPN ipsilateral to a unilateral pelvic nerve transection . (D,E): Symmetric upregulation GFR $\alpha 3-I R$ in the SPN on both sides following bilateral pelvic nerve transection. (F,G) GFR $\alpha 3$-IR axons formed dense clusters in the SPN but labeling was not see in somata. Scale bars represent $200 \mu \mathrm{m}$ (A), $50 \mu \mathrm{m}$ (B-E) and $50 \mu \mathrm{m}$ (F,G).

following experimental cystitis (Forrest and Keast, 2008), which should only affect a more restricted range of visceral afferents than those damaged by pelvic and hypogastric nerve transection. Our results suggest that there could be differences in how the central axons of somatic and visceral C-fiber sensory neurons respond to peripheral axotomy. Understanding these differences could provide useful understanding of biological mechanisms in somatic nociceptors that produce pathophysiology such as persistent post-surgical or neuropathic pain.

The effects of peripheral nerve injury on central terminals of C-fiber sensory neurons have been studied previously using labeling with isolectin B4-conjugates as a general marker of non-peptidergic projections (Bailey and Ribeiro-da-Silva, 

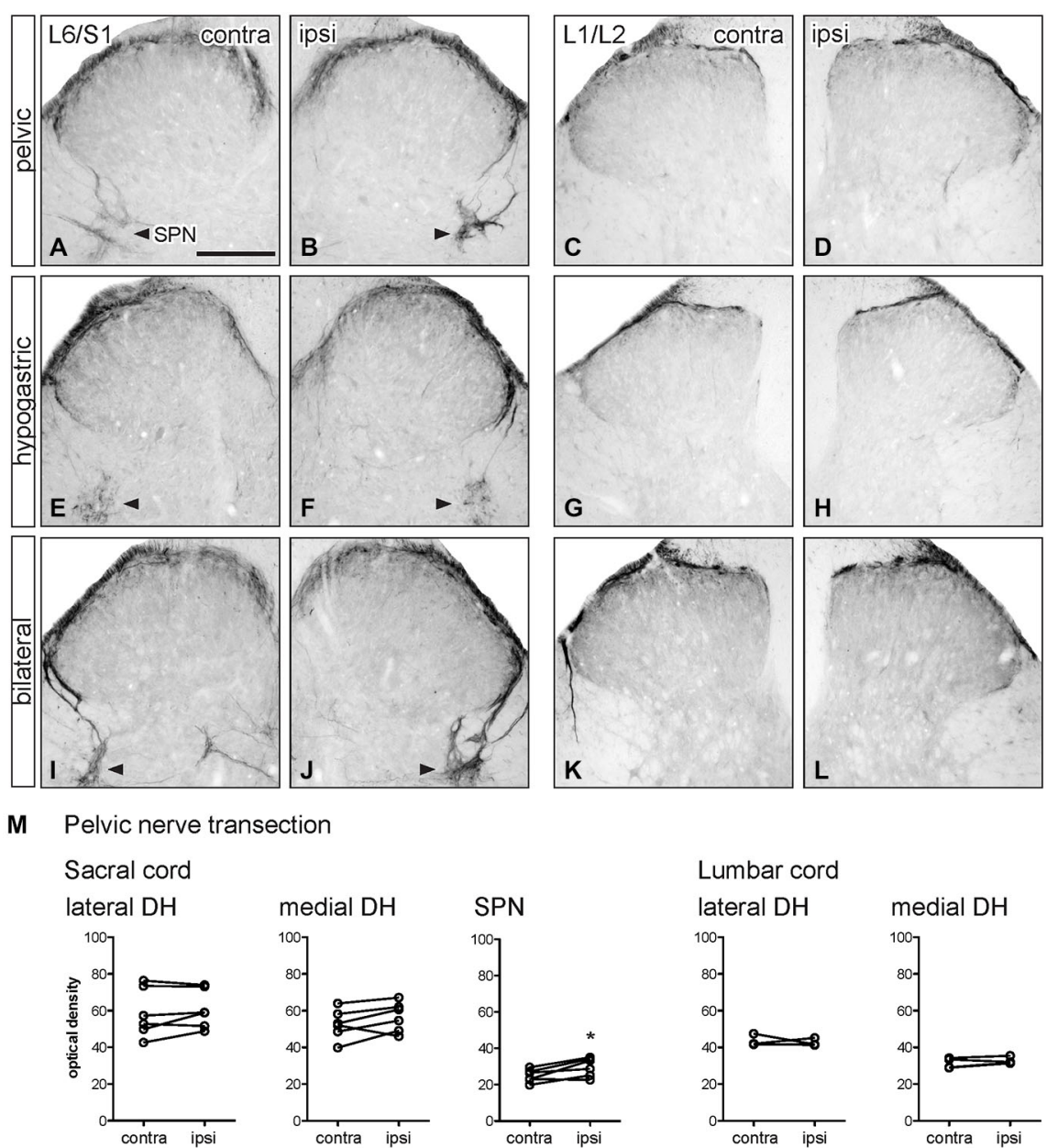

D
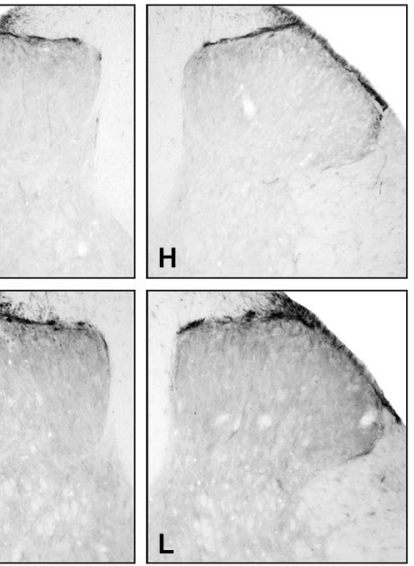

M Pelvic nerve transection

Sacral cord
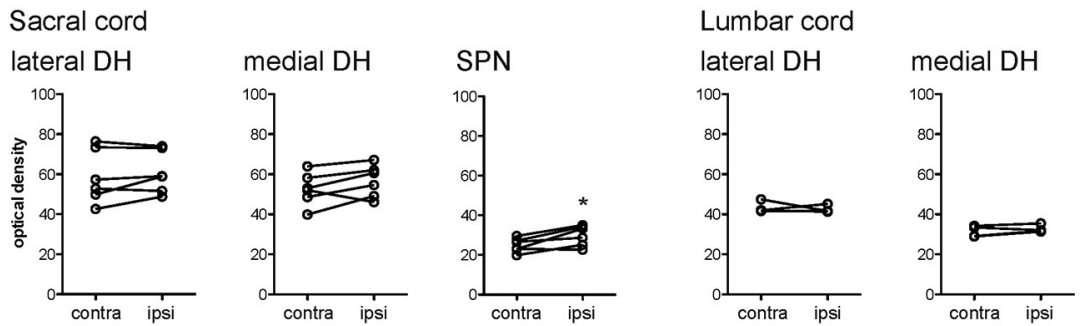

N Hypogastric nerve transection
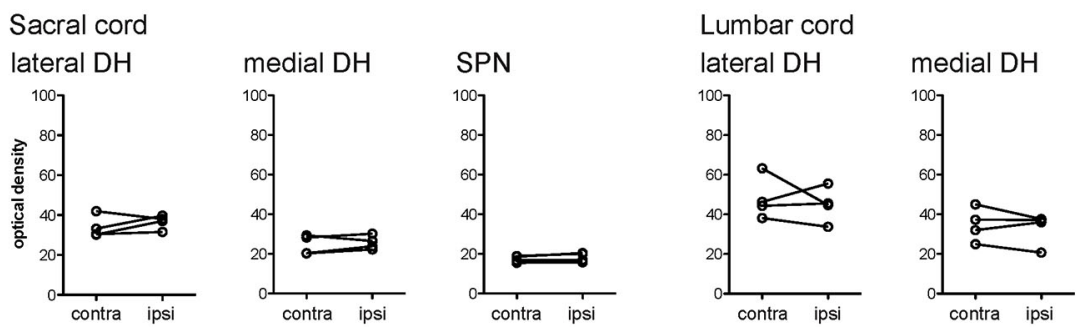

FIGURE 6 | Distribution of GFR $\alpha 3-I R$ in sacral and lumbar spinal cord following visceral nerve transection. For each type of injury, images were taken from left and right sides of the same section, and transverse segments of each spinal level from the same animal. (A-L) Images show GFRa3-IR in transverse sections of sacral (L6/S1) and lumbar (L1/2) dorsal horn at 7 days after unilateral pelvic nerve transection $(\mathbf{A}-\mathbf{D})$, unilateral hypogastric nerve transection $(\mathbf{E}-\mathbf{H})$ or bilateral pelvic and hypogastric nerve transection (I-L). (M) Following pelvic nerve transection, optical density analysis in sacral cord showed no changes in GFR $\alpha 3-I R$ in sacral dorsal horn, while an increase in GFR $\alpha 3-I R$ was seen in the sacral parasympathetic nucleus (SPN). In lumbar spinal cord, there was no effect of injury on GFR $\alpha 3-I R$. (N) Hypogastric nerve transection had no effect on GFR $\alpha 3-$ IR in sacral or lumbar spinal cord. Data represents the mean \pm SEM $(n=6$ rats for sacral data following unilateral pelvic nerve transection, $n=4$ rats for lumbar data following unilateral pelvic hypogastric nerve transection) and was analyzed using a paired $t$-test. The SPN in sacral cord is indicated with arrowheads (A,B,E,F,I,J). Scale bar represents $200 \mu \mathrm{m}$.
2006; Casals-Díaz et al., 2009) and CGRP-IR as a marker of peptidergic projections. However, in comparison to these general markers, our group has found that using GFR $\alpha 1-$, GFR $\alpha 2$ - and GFR $\alpha 3$-IR to identify subpopulations of each peptidergic and nonpeptidergic class provides greater anatomical resolution that reveals further specialization in the laminar projections from each population. This has also shown how each class responds independently and differently in rodent injury and inflammation 
TABLE 1 | Optical density (mean \pm SEM) of GDNF-family receptor alpha (GFR $\alpha$ )-immunoreactivity in sacral (L6/S1) and lumbar (L1/2) spinal cord segments following unilateral visceral nerve transection ${ }^{1}$.

\begin{tabular}{|c|c|c|c|c|c|c|c|c|}
\hline & & \multirow[b]{2}{*}{ Location of area of interest } & \multicolumn{3}{|c|}{ Pelvic nerve transection } & \multicolumn{3}{|c|}{ Hypogastric nerve transection } \\
\hline \multicolumn{2}{|c|}{ Immunoreactivity (spinal level) } & & Contralateral & Ipsilateral & $p$-value ${ }^{2}$ & Contralateral & Ipsilateral & $p$-value $e^{2,4}$ \\
\hline \multirow[t]{5}{*}{$\mathrm{GFR} \alpha 1$} & Sacral & Lateral dorsal horn & $57.2 \pm 6.9$ & $61.7 \pm 5.5$ & $0.07^{3}$ & $48.5 \pm 2.6$ & $46.8 \pm 2.5$ & 0.73 \\
\hline & & Medial dorsal horn & $50.3 \pm 7.5$ & $56.7 \pm 6.9$ & 0.04 & $42.3 \pm 1.8$ & $46.3 \pm 0.9$ & 0.23 \\
\hline & & SPN & $28.7 \pm 5.7$ & $36.2 \pm 4.5$ & 0.01 & $21.3 \pm 1.4$ & $22.2 \pm 1.7$ & 0.77 \\
\hline & Lumbar & Lateral dorsal horn & $57.3 \pm 3.0$ & $59.7 \pm 1.9$ & $0.36^{4}$ & $49.5 \pm 2.1$ & $52.2 \pm 3.4$ & 0.25 \\
\hline & & Medial dorsal horn & $53.9 \pm 3.5$ & $54.6 \pm 2.4$ & 0.75 & $51.1 \pm 2.8$ & $49.5 \pm 2.5$ & 0.45 \\
\hline \multirow[t]{4}{*}{ GFR $\alpha 2$} & Sacral & Lateral dorsal horn & $64.3 \pm 1.6$ & $64.3 \pm 1.9$ & $0.97^{3}$ & $49.8 \pm 4.1$ & $45.7 \pm 6.7$ & 0.50 \\
\hline & & Medial dorsal horn & $63.6 \pm 1.7$ & $64.3 \pm 2.4$ & 0.74 & $51.6 \pm 3.3$ & $49.4 \pm 4.3$ & 0.38 \\
\hline & Lumbar & Lateral dorsal horn & $66.1 \pm 2.7$ & $63.5 \pm 1.6$ & $0.38^{4}$ & $65.3 \pm 2.9$ & $63.7 \pm 2.8$ & 0.64 \\
\hline & & Medial dorsal horn & $59.9 \pm 2.3$ & $57.3 \pm 2.4$ & 0.02 & $60.3 \pm 1.8$ & $58.3 \pm 3.1$ & 0.45 \\
\hline \multirow[t]{5}{*}{ CGRP } & Sacral & Lateral dorsal horn & $39.7 \pm 5.3$ & $31.6 \pm 5.9$ & $0.01^{3}$ & $29.9 \pm 3.5$ & $32.1 \pm 3.6$ & 0.56 \\
\hline & & Medial dorsal dorn & $30.9 \pm 3.1$ & $29.0 \pm 3.5$ & 0.26 & $27.8 \pm 2.2$ & $30.4 \pm 3.5$ & 0.28 \\
\hline & & SPN & $16.8 \pm 1.3$ & $12.9 \pm 2.3$ & 0.12 & $15.9 \pm 2.1$ & $17.7 \pm 1.3$ & 0.28 \\
\hline & Lumbar & Lateral dorsal horn & $38.5 \pm 4.4$ & $31.8 \pm 2.2$ & $0.30^{4}$ & $32.9 \pm 2.8$ & $34.7 \pm 3.0$ & 0.38 \\
\hline & & Medial dorsal horn & $22.0 \pm 1.9$ & $20.7 \pm 1.2$ & 0.58 & $24.3 \pm 2.2$ & $23.4 \pm 1.7$ & 0.21 \\
\hline \multirow[t]{5}{*}{ GFR $\alpha 3$} & Sacral & Lateral dorsal horn & $58.7 \pm 5.5$ & $60.9 \pm 4.3$ & $0.29^{3}$ & $33.9 \pm 2.8$ & $36.6 \pm 1.8$ & 0.37 \\
\hline & & Medial dorsal horn & $52.6 \pm 3.4$ & $56.6 \pm 3.3$ & 0.13 & $24.6 \pm 2.5$ & $25.7 \pm 1.7$ & 0.47 \\
\hline & & SPN & $24.9 \pm 1.4$ & $29.8 \pm 2.1$ & 0.02 & $17.5 \pm 0.8$ & $18.3 \pm 1.2$ & 0.12 \\
\hline & Lumbar & Lateral dorsal horn & $43.3 \pm 1.4$ & $42.5 \pm 0.9$ & $0.69^{4}$ & $47.9 \pm 5.4$ & $44.9 \pm 4.5$ & 0.63 \\
\hline & & Medial dorsal horn & $31.4 \pm 1.4$ & $32.6 \pm 0.9$ & 0.27 & $34.8 \pm 4.2$ & $32.9 \pm 4.1$ & 0.49 \\
\hline
\end{tabular}

${ }^{1}$ For each rat a single estimate of the optical density expressed in arbitrary units was obtained by averaging measurements from six sections per rat.

${ }^{2}$ Ipsilateral vs. contralateral (Paired t-test). ${ }^{3} n=6 .{ }^{4} n=4$. Abbreviation: SPN, sacral parasympathetic nucleus.

models (Kalous et al., 2007, 2009; Forrest and Keast, 2008; Keast et al., 2010). However, it is important to note that our previous analyses of the lumbar (L4-L5) and lower thoracic (T9-T12) cord were aided by prior detailed reporting of the somatotopy of the sensory projections at these levels. By comparison, there is an incomplete understanding of somatotopy of the sensory input from the pelvic and hypogastric nerves in rat. HRP tracing has been used to identify sensory projections within transverse sections but not the rostrocaudal distribution of this projection across spinal cord segments. More detailed anatomical descriptions are available for cat (Morgan et al., 1981, 1986) but we are unaware of these identifying any clear somatotopic difference of the medial vs. the lateral dorsal horn.

Our analysis of the non-peptidergic type of C-fiber afferent found that GFR $\alpha 1$-IR increased locally in the medial dorsal horn of the sacral spinal cord after unilateral pelvic nerve transection. This was the only effect of visceral nerve injury on non-peptidergic C-fiber axons expressing GFR $\alpha 1$ - or GFR $\alpha 2$ IR in this study, although it is possible that this effect occurred in the myelinated class of GFR $\alpha 1$-IR axons. The localized effect of visceral nerve injury on afferent fibers expressing GFR $\alpha 1$ contrasts with the far more extensive increase in GFR $\alpha 1$-IR and localized reductions of GFR $\alpha 2$-IR that occur in the dorsal horn of the lumbar spinal cord following sciatic nerve injury (Keast et al., 2010). Following sciatic nerve injury, these changes are accompanied by an increase in GFR $\alpha$ l-IR neurons in lumbar DRG, demonstrating an upregulation of the receptor protein. These different outcomes could be significant in the context of the biological activity of the endogenous ligand, a potential source of which are DRG neurons that express GDNF mRNA (Kashiba et al., 2003) and protein (Holstege et al., 1998; Jongen et al., 1999) and release it from their central terminals. GDNF administration in spinal cord is reported to have potent analgesic activity in neuropathic pain models (Boucher et al., 2000).

Following sciatic nerve injury GFR $\alpha 2$-IR within lumbar cord decreases due to a reduction of primary afferents expressing this receptor (Keast et al., 2010). By contrast, we found GFR $\alpha 2$-IR was mostly unaffected by visceral nerve transection, except for a small reduction in GFR $\alpha 2$-IR in the medial dorsal horn of lumbar cord after pelvic nerve transection. This effect was unexpected as we are unaware of prior evidence for the lumbar spinal region receiving sacral afferent input. We believe the contrasting effects of sciatic and visceral nerve transection on GFR $\alpha 2$-IR in spinal cord could be explained by this class of C-fiber neuron having relatively limited projections to the pelvic viscera. This is suggested by our previous analyses that found very few GFR $\alpha 2$ IR sacral afferent neurons project to the rat bladder (Forrest and Keast, 2008; Forrest et al., 2013). The biological significance of this limited projection of GFR $\alpha 2$-IR sensory neurons to pelvic viscera has yet to be determined.

In this study, the only effect of visceral nerve injury on the peptidergic type of C-fiber afferents was a localized reduction in CGRP-IR in the lateral dorsal horn of sacral spinal cord after pelvic nerve transection. Around one third to half of all CGRP-IR peptidergic neurons also contain GFR $\alpha 3$ (Kalous et al., 2009; Forrest et al., 2013). Consistent with previous studies, GFR $\alpha 3$-IR fibers projected only to the more superficial of the laminae that receive input from CGRP-IR fibers overall, but these were not affected by visceral nerve transection. The location in which CGRP-IR was decreased corresponds with the projection patterns of sacral afferent axons entering the dorsal horn that travel via the pelvic nerve (Morgan et al., 1981). The localized 
reduction in CGRP-IR could therefore be explained by selective degeneration of CGRP-IR axons that are more susceptible to axotomy. As the axons expressing GFR $\alpha 3$ were not affected this could be consistent with evidence that suggest the endogenous ligand, artemin, has pro-regeneration effects on sensory axons in the spinal cord (Gardell et al., 2003; Wang et al., 2008).

Unlike the L4-L5 segments of the lumbar spinal cord, the sacral (L6-S1) and upper lumbar (L1-L2) spinal cord also contain autonomic preganglionic neurons that project in the corresponding pelvic and hypogastric nerves. These autonomic regions provide another target for $\mathrm{C}$-fiber input in addition to the dorsal horn. Therefore, C-fibers expressing the GDNF receptor, GFR $\alpha 1$, and the artemin receptor, GFR $\alpha 3$, are situated in close proximity to preganglionic neurons, many of which also express GFR $\alpha 1$ and GFR $\alpha 3$. The functional significance of this relationship is not known but it suggests GDNF and artemin could influence reflex functions by modulating sensoryautonomic interactions within the spinal cord. On this basis we analyzed the effects of visceral nerve injury in these spinal cord regions.

In uninjured rats, GFR $\alpha 1-I R$ afferents project via the LCP and terminate in the region of the SPN, where small numbers of parasympathetic preganglionic neurons are also GFR $\alpha 1$-IR (Forrest and Keast, 2008). Other spinal cord preganglionic neurons in the thoracic IML also express both Ret and GFR $\alpha 1$ mRNA (Schober et al., 1999) but the role of this signaling system in normal circuit function is not known. However, there is considerable evidence supporting a role for these receptors promoting survival of sympathetic preganglionic neurons after injury (Schober et al., 1999; Schober and Unsicker, 2001). In our study, visceral nerve injury upregulated GFR $\alpha 1$-IR in parasympathetic preganglionic neurons in the SPN. All parasympathetic preganglionic neurons innervating the pelvic ganglia are located in the SPN (Morgan et al., 1981) and are therefore axotomised by pelvic nerve transection. Visceral nerve injury also broadly increased GFR $\alpha 1$ - and GFR $\alpha 3$-IR in the SPN, but we could not determine if this was an effect on GFR $\alpha 1$-IR C-fiber afferent terminals or local recurrent

\section{References}

Airaksinen, M. S., and Saarma, M. (2002). The GDNF family: signalling, biological functions and therapeutic value. Nat. Rev. Neurosci. 3, 383-394. doi: 10. 1038/nrn812

Anand, P., Ghatei, M. A., Christofides, N. D., Blank, M. A., McGregor, G. P., Morrison, J. F., et al. (1991). Differential neuropeptide expression after visceral and somatic nerve injury in the cat and rat. Neurosci. Lett. 128, 57-60. doi: 10. 1016/0304-3940(91)90759-m

Bailey, A. L., and Ribeiro-da-Silva, A. (2006). Transient loss of terminals from non-peptidergic nociceptive fibers in the substantia gelatinosa of spinal cord following chronic constriction injury of the sciatic nerve. Neuroscience 138, 675-690. doi: 10.1016/j.neuroscience.2005.11.051

Boucher, T. J., Okuse, K., Bennett, D. L., Munson, J. B., Wood, J. N., and Mcmahon, S. B. (2000). Potent analgesic effects of GDNF in neuropathic pain states. Science 290, 124-127. doi: 10.1126/science.290.5489.124

Carlton, S. M., Mcneill, D. L., Chung, K., and Coggeshall, R. E. (1988). Organization of calcitonin gene-related peptide-immunoreactive terminals in the primate dorsal horn. J. Comp. Neurol. 276, 527-536. doi: 10.1002/cne. 902760407 axons projecting from preganglionic neurons. Other studies also provide evidence of neuroplasticity in these preganglionic neurons following visceral nerve injury. For example, many preganglionic neurons in the SPN that express GFR $\alpha 1$ also express NOS-IR (Forrest and Keast, 2008), which is upregulated after pelvic ganglionectomy (Vizzard et al., 1995). This contrasts with expression of the acetylcholine synthesizing enzyme, choline acetyltransferase (ChAT), which is downregulated in preganglionic neurons that express the injury markers c-Jun and ATF-3 after pelvic nerve transection (Peddie and Keast, 2011). The functional significance of the changes we observed in GFR $\alpha 1$ - and GFR $\alpha 3$-IR in the SPN has yet to be determined, but again the potential role of local sensory axons as a potential source of GDNF could be considered. This locally derived GDNF could promote regeneration of injured parasympathetic preganglionic neurons or increase neuronal excitability of pelvic visceral spinal cord pathways following injury (Boucher et al., 2000; Pezet and McMahon, 2006).

This study found no effect of hypogastric nerve transection on the $\mathrm{C}$-fiber sensory input or autonomic preganglionic neurons in the upper lumbar spinal cord. The hypogastric nerve contains sympathetic preganglionic axons that project to the pelvic ganglia from neurons located in L1/L2 spinal segments (Hancock and Peveto, 1979; Morgan et al., 1981). The preganglionic neurons projecting to pelvic ganglia via the hypogastric nerve are mostly (>80\%) located in the DGC and not the IML (Hancock and Peveto, 1979). Unlike the SPN in sacral spinal cord, the DGC receives minimal input from GFR $\alpha 1-$, GFR $\alpha 3$ - or CGRP-IR C-fiber afferents. There was not any upregulation of these markers following axotomy of lumbar afferent and sympathetic preganglionic axons in the hypogastric nerve.

\section{Acknowledgments}

This study was supported by Project Grants 1003512, 1022941 and Senior Research Fellowship 632903 from the National Health and Medical Research Council (NHMRC) of Australia.

Casals-Díaz, L., Vivó, M., and Navarro, X. (2009). Nociceptive responses and spinal plastic changes of afferent c-fibers in three neuropathic pain models induced by sciatic nerve injury in the rat. Exp. Neurol. 217, 84-95. doi: 10.1016/j.expneurol.2009. 01.014

Christie, K. J., and Zochodne, D. (2013). Peripheral axon regrowth: new molecular approaches. Neuroscience 240, 310-324. doi: 10.1016/j.neuroscience.2013. 02.059

Costigan, M., Scholz, J., and Woolf, C. J. (2009). Neuropathic pain: a maladaptive response of the nervous system to damage. Annu. Rev. Neurosci. 32, 1-32. doi: 10.1146/annurev.neuro.051508.135531

Ernsberger, U. (2008). The role of gdnf family ligand signalling in the differentiation of sympathetic and dorsal root ganglion neurons. Cell Tissue Res. 333, 353-371. doi: 10.1007/s00441-008-0634-4

Forrest, S. L., and Keast, J. R. (2008). Expression of receptors for glial cell linederived neurotrophic factor family ligands in sacral spinal cord reveals separate targets of pelvic afferent fibres. J. Comp. Neurol. 506, 989-1002. doi: 10. $1002 /$ cne. 21535

Forrest, S. L., Osborne, P. B., and Keast, J. R. (2013). Characterization of bladder sensory neurons in the context of myelination, receptors for pain modulators 
and acute responses to bladder inflammation. Front. Neurosci. 7:206. doi: 10. 3389/fnins.2013.00206

Forrest, S. L., Osborne, P. B., and Keast, J. R. (2014). Characterization of axons expressing the artemin receptor in the female rat urinary bladder: a comparison with other major neuronal populations. J. Comp. Neurol. 522, 3900-3927. doi: 10.1002/cne.23648

Gardell, L. R., Wang, R., Ehrenfels, C., Ossipov, M. H., Rossomando, A. J., Miller, S., et al. (2003). Multiple actions of systemic artemin in experimental neuropathy. Nat. Med. 9, 1383-1389. doi: 10.1038/nm944

Hamlin, A. S., McNally, G. P., and Osborne, P. B. (2007). Induction of c-fos and zif268 in the nociceptive amygdala parallel abstinence hyperalgesia in rats briefly exposed to morphine. Neuropharmacology 343, 330-344. doi: 10.1016/j. neuropharm.2007.05.017

Hancock, M. B., and Peveto, C. A. (1979). Preganglionic neurons in the sacral spinal cord of the rat: an hrp study. Neurosci. Lett. 11, 1-5. doi: 10.1016/03043940(79)90046-6

Holstege, J. C., Jongen, J. L., Kennis, J. H., van Rooyen-Boot, A. A., and Vecht, C. J. (1998). Immunocytochemical localization of gdnf in primary afferents of the lumbar dorsal horn. Neuroreport 9, 2893-2897. doi: 10.1097/00001756199808240-00039

Jensen, T., Baron, R., Haanpää, M., Kalso, E., Loeser, J. D., Rice, A. S. C., et al. (2011). A new definition of neuropathic pain. Pain 152, 2204-2205. doi: 10. 1016/j.pain.2011.06.017

Johansen, A., Romundstad, L., Nielsen, C. S., Schirmer, H., and Stubhaug, A. (2012). Persistent postsurgical pain in a general population: prevalence and predictors in the tromso study. Pain 153, 1390-1396. doi: 10.1016/j.pain.2012. 02.018

Jongen, J. L., Dalm, E., Vecht, C. J., and Holstege, J. C. (1999). Depletion of gdnf from primary afferents in adult rat dorsal horn following peripheral axotomy. Neuroreport 10, 867-871. doi: 10.1097/00001756-199903170-00036

Kalous, A., and Keast, J. R. (2010). Conditioning lesions enhance growth state only in sensory neurons lacking calcitonin gene-related peptide and isolectin b4binding. Neuroscience 166, 107-121. doi: 10.1016/j.neuroscience.2009.12.019

Kalous, A., Osborne, P. B., and Keast, J. R. (2007). Acute and chronic changes in dorsal horn innervation by primary afferents and descending supraspinal pathways after spinal cord injury. J. Comp. Neurol. 504, 238-253. doi: 10. $1002 /$ cne. 21412

Kalous, A., Osborne, P. B., and Keast, J. R. (2009). Spinal cord compression injury in adult rats initiates changes in dorsal horn remodeling that may correlate with development of neuropathic pain. J. Comp. Neurol. 513, 668-684. doi: 10. $1002 /$ cne. 21986

Kashiba, H., Uchida, Y., and Senba, E. (2003). Distribution and colocalization of ngf and gdnf family ligand receptor mrnas in dorsal root and nodose ganglion neurons of adult rats. Brain Res. Mol. Brain Res. 110, 52-62. doi: 10.1016/s0169$328 x(02) 00584-3$

Keast, J. R. (2006). Plasticity of pelvic autonomic ganglia and urogenital innervation. Int. Rev. Cytol. 248, 141-208. doi: 10.1016/s0074-7696(06)48003-7

Keast, J. R., Forrest, S. L., and Osborne, P. B. (2010). Sciatic nerve injury in adult rats causes distinct changes in the central projections of sensory neurons expressing different glial cell line-derived neurotrophic factor family receptors. J. Comp. Neurol. 518, 3024-3045. doi: 10.1002/cne.22378

Lawson, S. N., Perry, M. J., Prabhakar, E., and Mccarthy, P. W. (1993). Primary sensory neurones: neurofilament, neuropeptides and conduction velocity. Brain Res. Bull. 30, 239-243. doi: 10.1016/0361-9230(93)90250-f

Maas, C. P., Trimbos, J. B., Deruiter, M. C., van de Velde, C. J., and Kenter, G. G. (2003). Nerve sparing radical hysterectomy: latest developments and historical perspective. Crit. Rev. Oncol. Hematol. 48, 271-279. doi: 10.1016/s10408428(03)00122-7

Morgan, C., deGroat, W. C., and Nadelhaft, I. (1986). The spinal distribution of sympathetic preganglionic and visceral primary afferent neurons that send axons into the hypogastric nerves of the cat. J. Comp. Neurol. 243, 23-40. doi: 10.1002/cne.902430104

Morgan, C., Nadelhaft, I., and de Groat, W. C. (1981). The distribution of visceral primary afferents from the pelvic nerve to lissauer's tract and the spinal gray matter and its relationship to the sacral parasympathetic nucleus. J. Comp. Neurol. 201, 415-440. doi: 10.1002/cne.902010308

Nadelhaft, I., and Booth, A. M. (1984). The location and morphology of preganglionic neurons and the distribution of visceral afferents from the rat pelvic nerve: a horseradish peroxidase study. J. Comp. Neurol. 226, 238-245. doi: 10.1002/cne.902260207

Nadelhaft, I., and McKenna, K. E. (1987). Sexual dimorphism in sympathetic preganglionic neurons of the rat hypogastric nerve. J. Comp. Neurol. 256, 308-315. doi: 10.1002/cne.902560210

Navarro, X., Vivo, M., and Valero-Cabre, A. (2007). Neural plasticity after peripheral nerve injury and regeneration. Prog. Neurobiol. 82, 163-201. doi: 10. 1016/j.pneurobio.2007.06.005

Orozco, O. E., Walus, L., Sah, D. W., Pepinsky, R. B., and Sanicola, M. (2001). Gfralpha3 is expressed predominantly in nociceptive sensory neurons. Eur. J. Neurosci. 13, 2177-2182. doi: 10.1046/j.0953-816x.2001. 01596.x

Peddie, C. J., and Keast, J. R. (2011). Pelvic nerve injury causes a rapid decrease in expression of choline acetyltransferase and upregulation of c-jun and atf-3 in a distinct population of sacral preganglionic neurons. Front. Neurosci. 5:6. doi: 10.3389/fnins.2011.00006

Pezet, S., and McMahon, S. B. (2006). Neurotrophins: mediators and modulators of pain. Annu. Rev. Neurosci. 29, 507-538. doi: 10.1146/annurev.neuro.29. 051605.112929

Scheib, J., and Höke, A. (2013). Advances in peripheral nerve regeneration. Nat. Rev. Neurol. 9, 668-676. doi: 10.1038/nrneurol.2013.227

Schober, A., Hertel, R., Arumae, U., Farkas, L., Jaszai, J., Krieglstein, K., et al. (1999). Glial cell line-derived neurotrophic factor rescues target-deprived sympathetic spinal cord neurons but requires transforming growth factor-beta as cofactor in vivo. J. Neurosci. 19, 2008-2015.

Schober, A., and Unsicker, K. (2001). Growth and neurotrophic factors regulating development and maintenance of sympathetic preganglionic neurons. Int. Rev. Cytol. 205, 37-76. doi: 10.1016/s0074-7696(01)05002-1

Schug, S. A. (2012). Persistent post-surgical pain: a view from the other side of the fence. Pain 153, 1344-1345. doi: 10.1016/j.pain.2012.02.041

Sugiura, Y., Terui, N., and Hosoya, Y. (1989). Difference in distribution of central terminals between visceral and somatic unmyelinated (c) primary afferent fibers. J. Neurophysiol. 62, 834-840.

Sugiura, Y., Terui, N., Hosoya, Y., Tonosaki, Y., Nishiyama, K., and Honda, T. (1993). Quantitative analysis of central terminal projections of visceral and somatic unmyelinated (c) primary afferent fibers in the guinea pig. J. Comp. Neurol. 332, 315-325. doi: 10.1002/cne.903320305

Vizzard, M. A., Erdman, S. L., and de Groat, W. C. (1995). Increased expression of neuronal nitric oxide synthase (nos) in visceral neurons after nerve injury. $J$. Neurosci. 15, 4033-4045.

von Hehn, C. A., Baron, R., and Woolf, C. J. (2012). Deconstructing the neuropathic pain phenotype to reveal neural mechanisms. Neuron $73,638-652$. doi: 10.1016/j.neuron.2012.02.008

Walsh, P. C., and Donker, P. J. (1982). Impotence following radical prostatectomy: insight into etiology and prevention. J. Urol. 128, 492-497.

Wang, R., King, T., Ossipov, M. H., Rossomando, A. J., Vanderah, T. W., Harvey, P., et al. (2008). Persistent restoration of sensory function by immediate or delayed systemic artemin after dorsal root injury. Nat. Neurosci. 11, 488-496. doi: 10.1038/nn2069

Yang, Q., Wu, Z., Hadden, J. K., Odem, M. A., Zuo, Y., Crook, R. J., et al. (2014). Persistent pain after spinal cord injury is maintained by primary afferent activity. J. Neurosci. 34, 10765-10769. doi: 10.1523/jneurosci.531613.2014

Yoshimura, N. (1999). Bladder afferent pathway and spinal cord injury: possible mechanisms inducing hyperreflexia of the urinary bladder. Prog. Neurobiol. 57, 583-606. doi: 10.1016/s0301-0082(98)00070-7

Conflict of Interest Statement: The authors declare that the research was conducted in the absence of any commercial or financial relationships that could be construed as a potential conflict of interest.

Copyright (c) 2015 Forrest, Payne, Keast and Osborne. This is an open-access article distributed under the terms of the Creative Commons Attribution License (CC BY). The use, distribution and reproduction in other forums is permitted, provided the original author(s) or licensor are credited and that the original publication in this journal is cited, in accordance with accepted academic practice. No use, distribution or reproduction is permitted which does not comply with these terms. 